\title{
Multimodal Analysis of Diamond Crystals and Layers Using RISE Microscopy
}

\author{
Rostislav Váňa ${ }^{1}$, Jiří Dluhoš ${ }^{1}$, Marián Varga ${ }^{2}$, Christoph $\mathrm{Schmid}^{3}$, and Alexander Kromka ${ }^{2}$ \\ 1. TESCAN Brno, s.r.o, Brno, Czech Republic. \\ 2. Institute of Physics, Czech Academy of Sciences, v.v.i., Prague 6, Czech Republic. \\ 3. Department of Materials Science, TU Darmstadt, Darmstadt, Germany.
}

Diamond is a material that combines several useful intrinsic properties such as a wide bandgap, the highest mechanical hardness, broad optical transparency (from UV through IR region), resistance to most chemical treatments, the highest thermal conductivity at room temperature and controllable surface termination at the molecular level. Moreover, diamond is also a biocompatible material because it is carbonaceous. Thanks to these properties, mono- and poly-crystalline diamonds (PCD) are being intensively studied for uses in micro- or bio-electronics, sensing and drug delivery.

Furthermore, optically tailored diamond films and nanostructures have attracted great attention due to their remarkable properties suitable for emerging technologies such as photonics, integrated quantum photonics and quantum computing. However, the properties of diamond have to be controlled at the nano- and micro-scopic scales, and this is not possible without advanced analytical techniques.

Here we present the applicability of Raman Integrated Scanning Electron (RISE) microscopy [1] for diamond characterization. The combination of a TESCAN UHR SEM column - Triglav ${ }^{\text {TM }}$ and an integrated Confocal Raman Microscope (CRM) within one vacuum chamber enables simple movement between these two methods of inspection. The UHR SEM is ideal for low-kV morphological observation of diamond films and grains, whereas Raman and photoluminescence (PL) imaging substantially extend the outcome from one instrument. The resolution of CRM $-360 \mathrm{~nm}$ with the 532 $\mathrm{nm}$ green laser - makes the RISE system exemplary for characterizing individual diamond grains or continuous films. Moreover, an area of $250 \times 250 \mu \mathrm{m}^{2}$ can be imaged at this resolution in a single scan of Raman or PL mapping. Additionally, utilizing the built-in Focused Ion Beam (FIB) enables in-situ modification of the samples, thus enhancing the processing/depth analysis of the material.

The main difference between mono- and poly-crystalline diamonds ( $\mathrm{sp}^{3}$ hybridization) is the absence or presence of the $\mathrm{sp}^{2}$ bonded carbon phases respectively. Raman spectroscopy gives very precise information on the type of carbon hybridization $\left(\mathrm{sp}^{3}\right.$ versus $\left.\mathrm{sp}^{2}\right)$ and on its structural quality and order. The Raman spectrum of mono-diamond is characterized by a sharp peak at $1332 \mathrm{~cm}^{-1}$ (FWHM $=$ $\sim 1.2 \mathrm{~cm}^{-1}$ ). The Raman spectrum of PCD (Figure $1 \mathrm{~b}$ ) exhibits a broadening of the characteristic peak of diamond and comprises three additional peaks. The peak at around $1620 \mathrm{~cm}^{-1}$ is attributed to nondiamond carbon phases, and the peaks at $1150 \mathrm{~cm}^{-1}$ and $1450 \mathrm{~cm}^{-1}$ are attributed to trans-polyacetylene ( $t$-PA) chains located at the grain boundaries [2]. The RISE microscope allows the visualization of the $\mathrm{sp}^{2} / \mathrm{sp}^{3}$ carbon ratio distribution at high resolutions.

Intentional doping of diamond with other atoms (e.g. nitrogen, silicon, chromium or erbium) can produce a range of color centers with strong and stable fluorescence, long quantum coherence times and no photobleaching [3]. Especially, the spectral properties of Si-related color centers are promising due to their narrow, bright and stable single-photon emission. Their emission is situated in the red spectral region where the background diamond fluorescence is low [4]. Figure 1c shows a representative SEM micrograph of a single diamond grain imaged with topographical BSE contrast. The diamond grains 
were grown using a focused plasma chemical vapor deposition system under conditions where direct doping by $\mathrm{Si}$ atoms is possible [5]. The optical activity of the incorporated $\mathrm{SiV}$ centers in individual diamond grains was determined from high-resolution PL mapping. The overlaid PL map ( $738 \mathrm{~nm})$ with the corresponding SEM image is shown in Figure 1d. Different PL emission intensities are clearly identified which we attributed to different diamond grain shapes.

Several techniques for monitoring residual stress in diamond coatings were studied as part of the European FP7 project iSTRESS [6]. A Focused Ion Beam (FIB) was used for milling a double slot or other pre-determined geometry in diamond films. Within that processed area the residual stress is fully released and can be used as a strain-free reference for strain mapping using digital image correlation (FIB-DIC) and ex-situ [7] or in-situ CRM [by monitoring the Raman shift of $1332 \mathrm{~cm}^{-1}$ peak (Figure 1e, f)]. In summary, we demonstrate the unique value of the RISE microscope as an in-situ multimodal analytical workstation for identifying morphological variation, chemical purity, stress distribution or photoluminescence activity in the same sample area [8].

References:

[1] J. Jiruše et al, Journal of Vacuum Science \& Technology B 32 (2014) p. 6.

[2] M. Varga et al, Carbon 111 (2017), p. 54.

[3] J. Cajzl et al, Phys. Chem. Chem. Phys., (2017)

[4] E. Neu et al, Appl. Phys. Lett. 98 (2011), p. 243107.

[5] S. Potocky et al, Phys. Stat. Solidi B 252 (2015), p. 2580.

[6] iSTRESS Project website, http://www.istress.eu

[7] F. Ahmed et al, Surf. Coatings Technol. 237 (2013), p. 255.

[8] This work was supported by the MSMT project No. LD15003 within the frame of the COST Action MP1403 "Nanoscale Quantum Optics".

a)

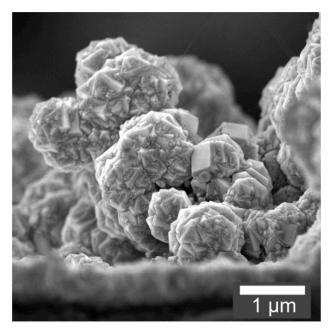

b)

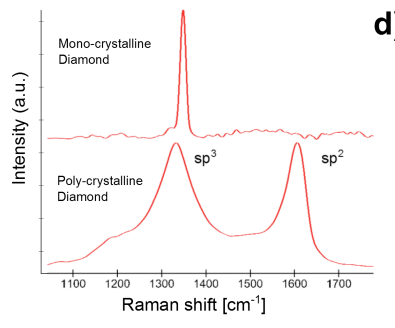

c)

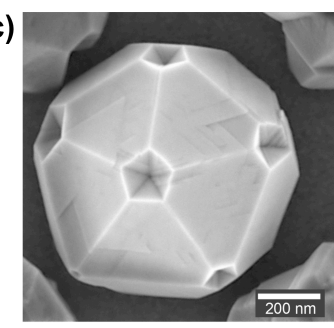

d)

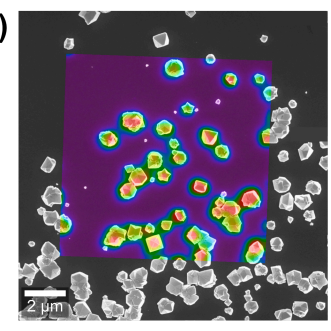

e)

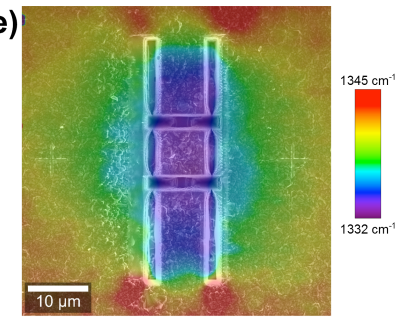

f)

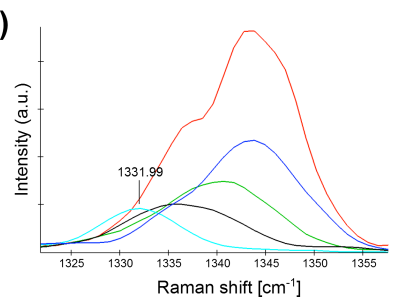

Figure 1. a) SEM image of a polycrystalline diamond film. b) Comparison of Raman spectra of monoand poly-crystalline diamond. c) SEM micrograph of a single diamond grain imaged with topographical BSE contrast. d) Overlay of the SEM image with SiV color center photoluminescence intensity measured by RISE microscope. e) Raman map of the strain released in PCD film milled by FIB superimposed on the SEM image. f) The corresponding Raman spectra of the diamond peak position. 